\title{
Fibre Products of Superelliptic Curves and Codes Therefrom
}

\author{
Serguei A. Stepanov and Ferruh Özbudak \\ Serguei A. Stepanov: Dept. of Mathematics, Bilkent University, 06533 Ankara, Turkey and \\ Steklov Mathematical Institute, Vavilov st. 42, Moscow GSP-1, 117966 Russia \\ Email: stepanov@fen.bilkent.edu.tr \\ Ferruh Özbudak: Dept. of Mathematics, Bilkent University, 06533 Ankara, Turkey \\ Email ozbudak@fen.bilkent.edu.tr
}

Our purpose is to construct new families of smooth projective curves over a finite field $F_{q}$ with a lot of $F_{q}$-rational points points. The genus in every such family is considerably less than the number of rational points, so the corresponding geometric Goppa codes have rather good parameters.

Let $X$ be a smooth projective curve of genus $g=g(X)$ defined over a finite field $F_{q}$. The Goppa construction of linear $[n, k, d]_{q}$-codes associated to the curve $X$ can be briefly described as follows. Let $\left\{x_{1}, \ldots, x_{n}\right\}$ be a set of $F_{q}$-rational points on $X$ and

$$
D_{0}=x_{1}+\cdots+x_{n} .
$$

Let $D$ be a $F_{q}$-rational divisor on $X$ such that Supp $D_{0} \cap$ Supp $D=\emptyset$, and $F_{q}(X)$ the field of rational functions on $X$. Consider the following vector space over $F_{q}$ :

$$
L(D)=\left\{f \in F_{q}(X)^{*} \mid(f)+D \geq 0\right\} \cup\{0\} .
$$

The linear $[n, k, d]_{q}$-code $C=C\left(D_{0}, D\right)$ associated to the pair $\left(D_{0}, D\right)$ is the image of the linear evaluation map

$$
\text { Ev : } L(D) \rightarrow F_{q}^{n}, \quad f \mapsto\left(f\left(x_{1}\right), \ldots, f\left(x_{n}\right)\right) .
$$

Such a $q$-ary linear code is called a geometric Goppa code. If $\operatorname{deg} D<n$, the map $E v$ is an injection, so $C \simeq L(D)$. It follows from the Riemann-Roch theorem that the relative parameters $R=k / n$ and $\delta=d / n$ of the code $C$ satisfy

$$
R \geq 1-\delta-\frac{g-1}{n} .
$$

In order to produce a family of asymptotically good geometric Goppa codes (when $n \rightarrow \infty$ ) for which $R+\delta$ comes above the Gilbert-Varshamov bound

$$
R \geq 1-H_{q}(\delta)
$$

one needs a family of smooth projective curves with a lot of $F_{q}$-rational points compared to the genus. Examples of such families are provided by modular curves (Ihara, TsfasmanVladut-Zink, C. Moreno), by Drinfeld modular curves (Tsfasman), and by Artin-Schreier coverings of the projective line $\mathbb{P}\left(F_{q}\right)$ (Garcia-Stichtenoth). As a result, one can construct an infinite sequence of geometric Goppa codes $C_{i}$ over $F_{q}(q$ is a square), which gives the lower bound

$$
R \geq 1-\delta-(\sqrt{q}-1)^{-1} \text {. }
$$

The line $R=1-\delta-(\sqrt{q}-1)^{-1}$ intersects the curve $R=$ $1-H_{q}(\delta)$ for $q \geq 49$.

Our purpose is to construct rather long geometric Goppa codes coming from fibre products of superelliptic curves $X_{s}$ given over $F_{q}$ by equations

$$
z_{i}^{\mu}=f_{i}(u), \quad 1 \leq i \leq s,
$$

where $f_{i}(u)$ are pairwise coprime polynomials of the same degree $m \geq 1$. We can exactly find a basis of the space of regular differential forms on $X_{s}$. This gives an easy way to calculate the genus of the smooth projective curve $X_{s}$. For example, if $\mu=2$ and the polynomials $f_{i}(u), 1 \leq i \leq s$, are square-free, we have

$$
g(X)=\left\{\begin{array}{lll}
(m s-3) 2^{s-2}+1 & \text { if } m \equiv 1 \quad(\bmod 2) \\
(m s-4) 2^{s-2}+1 & \text { if } m \equiv 0 \quad(\bmod 2)
\end{array}\right.
$$

On the other hand, we can choose the polynomials $f_{i}(u)$ in such a way to provide a lot of $F_{q}$-rational points on $X_{s}$. So, if $\mu=2, q=p^{\nu}\left(p=\operatorname{char} F_{q}>2\right)$, then for some special polynomials $f_{i}(u), 1 \leq i \leq s$, the number $N_{q}=N_{q}\left(X_{s}\right)$ of $F_{q}$-rational points on $X_{s}$ satisfies

$$
N_{q} \geq\left\{\begin{array}{cll}
\left(2 q^{1 / 2}-s\right) q^{1 / 2} 2^{s-1} & \text { if } \nu \equiv 0 & (\bmod 2) \\
2^{s} q & \text { if } \nu \equiv 1 & (\bmod 2)
\end{array}\right.
$$

Setting $n=N_{q}$ and using the Goppa construction we obtain

$$
R \geq 1-\delta-\frac{s q^{1 / 2}-3}{2\left(2 q^{1 / 2}-s\right) q^{1 / 2}}, \quad \text { for } \quad \nu \equiv 0 \quad(\bmod 2)
$$

and

$$
R \geq 1-\delta-\frac{s q^{1 / 2}-4}{4 q}, \text { for } \nu \equiv 1 \quad(\bmod 2) .
$$

Unfortunately, the parameter $s$ in our construction is bounded by $q^{1 / 2}$, and as a result the genus $g=g\left(X_{s}\right)$ is bounded by

$$
(q-3) 2^{\sqrt{q}-2}+1 \text {. }
$$

However, since the above upper bound is large enough for $q \geq q_{0}$, the curves $X_{s}$ provide suffuciently long geometric Goppa codes with rather good parameters. Moreover, these codes have very easy construction and decoding algorithms. We note also, that this approach, being extended to the case $\boldsymbol{\mu}>2$, gives a possibility to construct rather good linear codes in arbitrary characteristic $p \geq 2$ (sometimes with better parameters than in the case $\mu=2$ ). 